\title{
Reseña del XLV Congreso Anual Colegio Mexicano de Medicina Crítica
}

\author{
Dr. Jean Paul Vázquez Mathieu*
}

\section{Estimados miembros del COMMEC}

En esta ocasión, el Consejo Directivo decidió que la ciudad de Puebla de los Ángeles - histórica desde su fundación en 1531 - albergara el XLV Congreso Anual del Colegio Mexicano de Medicina Crítica, el XLII Congreso de las Enfermeras Especializadas en Medicina Crítica y Terapia Intensiva y la Primera Reunión del Colegio de Medicina Crítica de Puebla. Durante cinco días de intensa actividad académica para médicos y enfermeras, cursos, simposios y talleres llenaron un programa muy ambicioso con temas de actualidad presentados por expertos y la posibilidad de una interacción con los mismos.

Continuidad, calidad, calidez y excelencia fueron algunas de las principales características de nuestro congreso el pasado mes de octubre. Continuidad por seguir trabajando en el plan estratégico «Quo vadis» establecido hace ya varios años. Excelencia por los logros académicos fomentados, por la integración nacional e internacional de los médicos especialistas en medicina crítica y especialidades afines. Calidad por las extraordinarias ponencias de más de 28 profesores internacionales, 150 profesores nacionales y cerca de 50 profesores de enfermería. Calidez por la bienvenida que nos dio a todos los participantes la comisión de Puebla, así como su hermosa ciudad, localizada geográficamente en el centro entre Veracruz y la Ciudad de México, con una arquitectura que abarca épocas desde el Renacimiento hasta el Barroco neoclásico.

Fueron 12 los cursos precongreso para médicos y dos para enfermeras los días 01 y 02 de octubre, los cuales tuvieron una buena asistencia. Este año se diseñaron cuatro nuevos cursos: uno de ellos sobre sepsis, dos de simulación y crisis resource management (CRM), y el último de enfoque multimodal de la disfunción orgánica. Cabe mencionar que los cursos de «Fundamentos en Terapia Nutricional en el Enfermo Grave» y «Sepsis» fueron avalados en conjunto con la FEPIMCTI (Federación Panamericana e Ibérica de Terapia Intensiva), y todos, a su vez, avalados y reconocidos por nuestro colegio.

El día martes 02 de octubre tuvo lugar la ceremonia de inauguración, galardonada por la presencia de personalidades de la medicina crítica a nivel mundial, entre ellas, la Dra. Janice Zimmerman, presidenta de la Fe-

\footnotetext{
* Comisión Directiva. COMMEC 2017-2018.
}

Este artículo puede ser consultado en versión completa en http://www.medigraphic.com/medicinacritica deración Mundial de Sociedades de Medicina Crítica y Terapia Intensiva (WFSICCM), el Dr. Néstor Raimondi, presidente de la Federación Panamericana e Ibérica de Medicina Crítica y Terapia Intensiva (FEPIMCTI), el Dr. Jerry Zimmerman, presidente de la SCCM, la Dra. Mari Cruz Martín Delgado, presidenta de la SEMICYUC, y las personalidades la mesa directiva actual de nuestro colegio, así como de la filial de Puebla.

EI Dr. Ulises W. Cerón Díaz, presidente del COMMEC, dirigió a los asistentes unas palabras de bienvenida en donde explicó que después de un año de arduo trabajo con las diferentes comisiones y comités, se logró estructurar un programa académico de alto nivel; deseó a todos los asistentes que disfrutaran del congreso y de la hermosa ciudad de Puebla. La Dra. Janice Zimmerman, en un perfecto español, fue la encargada de inaugurar el congreso. En esta ocasión, la conferencia magistral le correspondió al Dr. Jerry Zimmerman, caracterizado por su lema «Less is more». Nos brindó una conferencia de muy elevado nivel académico y recordó que el uso adecuado de recursos en la enfermedad crítica, las tendencias actuales de sedar menos, movilidad temprana y el empoderamiento de la familia son puntos cruciales para una atención de calidad y seguridad.

Un concierto de ópera \& rock de Sierra-Roja fue el colofón de la ceremonia de inauguración, en donde nos hicieron recordar y vibrar todos esos grandes éxitos, interpretando desde obras como «Granada» hasta rock con «The Wall» de Pink Floyd.

Las sesiones plenarias, los simposios por parte del COMMEC y la industria, además de los talleres - algunos de ellos con modelo experimental- realizados durante el congreso se destacaron por el alto nivel académico y experiencia en el campo de todos los profesores. La gran demanda, la concurrencia a los mismos y el reconocimiento por parte de los asistentes se pudieron observar y constatar por los comentarios que se escuchaban en los pasillos del Centro Expositor de la Ciudad de Puebla de los Ángeles. Por segundo año, se realizó un simposio en conjunto con la Sociedad de Pediatría, el cual tuvo como profesor invitado al Dr. Jerry Zimmerman, pediatra y presidente de la SCCM.

La modalidad de presentación de los trabajos libres - durante la cual el jurado y los asistentes intercambiaban opiniones e intervenían haciendo comentarios ad hoc para los jóvenes y entusiastas investigadores participantes - fue muy bien recibida por todos. Este año se inscribieron más de 100 trabajos libres, lo que nos 
habla de un mayor entusiasmo por parte de los médicos jóvenes en hacer investigación y publicar en nuestra revista Medicina Crítica, cuyo editor en jefe es el Dr. José Javier Elizalde. Hacemos un agradecimiento extenso y felicitamos a los organizadores de los trabajos libres y a los profesores por su participación en la revisión y evaluación de los mismos en este año.

Durante el congreso se tuvieron reuniones de trabajo con las filiales, los diferentes capítulos y comisiones, así como la FEPIMCTI, aprovechando nuestro congreso para reuniones de trabajo y continuar con el plan estratégico iniciado el año pasado en la ciudad de Querétaro.

Debo mencionar que la perseverancia y el trabajo incansable por parte de la Comisión de Educación, encabezada por el Dr. Alfredo F. Sierra Unzueta, maestro de muchas generaciones, hizo posible la tercera reunión consecutiva con los profesores titulares y adjuntos de las diferentes sedes universitarias del país, con la finalidad de presentar los avances trabajados en relación con el PUEM. Gracias a todos los involucrados por intentar hacer más fuerte y unida a la medicina crítica mexicana.

A lo largo del congreso, durante algunos simposios y en el curso precongreso, la Dra. Rosario Muñoz nos pintó de verde, explicando e impulsando el proceso de donación y trasplante de órganos y tejidos de origen cadavérico en México. Nuevamente, muchas felicidades, Dra. Muñoz.

Por cuarto año consecutivo, tuvo lugar el Tazón Alvéolo, concurso de ventilación mecánica 2018, patrocinado por Medtronic, en donde participaron algunas sedes universitarias. El jurado estuvo integrado por profesores nacionales e internacionales de gran experiencia, quienes formularon preguntas en forma de "Jeopardy». En esta ocasión, la sede ganadora fue el Hospital Español de México. Felicidades.

El viernes, durante la ceremonia de clausura, el Dr. Elizalde hizo la entrega de premios a los tres mejores trabajos de investigación. El premio Mario Shapiro al mejor trabajo presentado fue para el Centro Médico ABC y los autores, Dra. Ahtziri Yunuén Rodríguez Santos, Dra. Andrea Rugenio Cabrera, Dr. Julio Sausa Sosa y sus colaboradores, por el trabajo titulado: «Asociación de mortalidad, deformación longitudinal global del ventrículo izquierdo y deformación circunferencial del ventrículo izquierdo en pacientes con sepsis en la unidad de terapia intensiva». El segundo lugar fue para el Hospital de Especialidades Núm. 14, UMAE 189, IMSS Veracruz, y los autores, Dr. Samuel Pascual Epigmenio, Dr. Jesús Salvador Sánchez Díaz, Dra. Karla Gabriela Peniche Moguel, Dr. Enrique Antonio Martínez Rodríguez, Dr. Josué Eli Villegas Domínguez y la Dra. María Verónica Calyeca Sánchez, por el trabajo: «Evaluación de la perfusión tisular en pacientes con choque séptico normodinámico versus hiperdinámico». El tercer lugar fue para el IMSS de León, Guanajuato, y los autores, Dra. Alma Érika Hernández Plata, Dra. Ma. Natalia Gómez González, Dr. Raúl Soriano Orozco y su grupo, por el trabajo titulado: «Relación entre la medición sonográfica de los músculos recto femoral y vasto intermedio con parámetros bioquímicos convencionales para valorar el estado nutricional en la unidad de cuidados intensivos». Muchas felicidades a los tres ganadores.

Por primera ocasión, este año se otorgó el premio Dr. Alberto Villazón Sahagún a un médico especialista en medicina crítica, colegiado joven con una trayectoria destacada en la parte asistencial, docente, de administración e investigación de la medicina crítica mexicana. Dicho reconocimiento fue otorgado al Dr. Silvio Antonio Ñamendys Silva de manos del Dr. Ulises W. Cerón Díaz. Muchas felicidades, Dr. Ñamendys, por su extraordinaria labor con nuestros enfermos.

EI Dr. Ulises Cerón, presidente actual del COMMEC, dio unas palabras de agradecimiento a todos los asistentes, profesores e industria por su invaluable participación durante el XLV Congreso Anual del Colegio Mexicano de Medicina Crítica.

El Dr. Esteban felicitó a los organizadores de este congreso y a la medicina crítica mexicana; lo cito: «Los felicito por el presente espléndido que tienen y el ilusionante futuro que parece que hace prever lo que tenemos y lo que se ha recorrido en estos años. Declaro concluido el LXV Congreso Anual del Colegio Mexicano de Medicina Crítica, LXII Congreso de las Enfermeras Especializadas en Medicina Crítica y Terapia Intensiva, y la Primera Reunión del Colegio de Medicina Crítica de Puebla».

La cena de clausura se encontró amenizada por un grupo musical que nos hizo recordar, al ritmo de sus canciones, diferentes épocas, motivo de risas y de compartir unos minutos con los amigos, maestros y compañeros después de una semana de gran nivel académico.

Se entregaron varios premios como libros, dos viajes todo pagado a Puerto Vallarta y el gran premio, un coche; el ganador fue el Dr. César Cruz Lozano de Tampico. Muchas felicidades.

Para finalizar, queremos agradecer a toda la industria por una participación sobresaliente con 36 stands y varios simposios, sin olvidar el patrocinio de asistentes y profesores. Esto, sin lugar a duda, contribuyó al éxito de este evento, por lo que esperamos contar nuevamente con todos ustedes el próximo año 2019 en la ciudad de León, Guanajuato. Con su asistencia y participación será, una vez más, un éxito.

Aprovecho, en nombre del COMMEC, para desearles un muy feliz año lleno de expectativas, gran producción científica y grandes logros. 